\title{
Diferencias en rendimiento académico según los niveles de las es- trategias cognitivas y de las estrategias de autorregulación
}

\section{Differences in academic achievement according to the levels of cognitive and of self-regulation strategies}

\author{
Antonio Valle ${ }^{1}$, Susana Rodríguez, Ramón G. Cabanach \\ Universidad de A Coruña, España \\ José C. Núñez, Julio A. González-Pienda \\ Universidad de Oviedo, España \\ Pedro Rosário \\ Universidad de Minho, Portugal
}

(Recepción: Septiembre 2009 - Aceptación: Diciembre 2009)

\begin{abstract}
Resumen
Entendiendo el estudio como una actividad estratégica y autorregulada y tomando como referencia la diferenciación entre estrategias cognitivas y estrategias de autorregulación realizada por diversos autores, en este trabajo se pretende comprobar si diferencias en el uso de estos tipos de estrategias conllevan niveles distintos de rendimiento académico. Los datos han sido recogidos a partir de 447 estudiantes de los cuatro cursos de la Enseñanza Secundaria Obligatoria (12 a 16 años, aproximadamente) correspondientes al Sistema Educativo Español. Han sido aplicados instrumentos de evaluación de estrategias cognitivas y de autorregulación y se han observado los logros académicos en diferentes áreas curriculares (matemática, lengua española, lengua inglesa, ciencias sociales y naturales, y música). Los resultados obtenidos indican nítidamente que cuanto mayor es el uso de estrategias cognitivas y de autorregulación, mayor es también el rendimiento académico en las áreas curriculares evaluadas.
\end{abstract}

Palabras clave: Estrategias cognitivas, aprendizaje autorregulado, rendimiento académico.

\begin{abstract}
Understanding study as a strategic and self-regulated activity and having in mind the distinction between cognitive and self-regulated learning strategies suggested in the literature, this paper analyses whether the differences in the use of this kind of strategies leads to different levels of academic achievement. Data were collected using a sample of 447 (12 to 16 years-old) students from Spanish Secondary Compulsive Education. Various instruments were applied to assess students' cognitive and self-regulated learning strategies. Students' marks in Maths, Spanish, English (Foreign language), Science, Social studies and Music were taken as indicators of academic achievement. Data suggest that the more students use cognitive and self-regulated learning strategies in a specific subject the better their level of achievement in that same subject.

Key words: Cognitive strategies; self-regulated learning; academic achievement.
\end{abstract}

1 Correspondencia: Antonio Valle Arias, Departamento de Psicología Evolutiva y de la Educación, Universidad de A Coruña. Campus de Elviña s/n, 15071 A Coruña. E-mail: vallar@udc.es 
Un número importante de estudiantes, especialmente aquellos que obtienen un bajo rendimiento, afirman encontrar dificultades para estudiar y, además, muchos de sus profesores suelen coincidir en esa problemática. Estos estudiantes pierden tiempo y esfuerzo sin darse cuenta siquiera de qué hacer para afrontar correctamente las actividades establecidas. No parecen capaces de reflexionar adecuadamente sobre lo que para ellos implica estudiar, mostrando dificultades para controlar y evaluar su proceso de aprender, justamente el desarrollo de esta conciencia metacognitiva parece el primer paso para posibilitar que el estudiante asuma mayor responsabilidad en sus aprendizajes. En consecuencia, estos alumnos tienen serias dificultades para organizar su conducta de estudio y para utilizar estrategias eficaces para aprender (Núnez, Solano, Pienda \& Rosário, 2006; Rosário, Mourão, Núñez, González-Pienda, Solano \& Valle, 2007).

Sin embargo, también es cierto que hay otros muchos alumnos que saben qué hacer y cuándo hacerlo, es decir, son capaces de interpretar sus experiencias pasadas de estudio de una forma significativa y tienen un buen conocimiento metacognitivo acerca de los procesos personales implicados en cada actividad. Estos estudiantes manifiestan, aunque no siempre son totalmente conscientes de ello, una teoría personal sobre el estudio que les permite mantener en su actividad diaria una conducta de estudio bien ajustada, conducta que parecen haber desarrollado por sí solos sin que nadie se haya dedicado a enseñarles las formas eficaces para estudiar (Rosário, Mourão, Soares, Chaleta, Grácio, Núnez \& González-Pienda, 2005; Zimmerman, 2002).

Cada vez es más necesario que los estudiantes aprendan a ser estratégicos, capaces de actuar intencionalmente para conseguir los objetivos de aprendizaje que se planteen, teniendo en cuenta las características de la tarea, las exigencias y demandas del entorno y las propias limitaciones y recursos de los que personalmente disponen. De esta forma, tanto en el ámbito académico como en el laboral, se hace necesario adaptar de forma estratégica las habilidades y los conocimientos adquiridos a un mundo con demandas diversas que exige de sus estudiantes y trabajadores supervisar lo que están haciendo obligándoles a ir más allá del dominio de rutinas automatizadas.

Pero la puesta en marcha de una actuación estratégica depende de las habilidades de control y regulación autónoma: la autorregulación del aprendizaje. Así, parece probado que la mejora permanente del aprendizaje requiere que los estudiantes sean gestores activos de sus conocimientos, estrategias, y demás recursos, es decir, sean capaces, por ejemplo, de estudiar los contenidos de las materias, hacer planes y coordinar recursos; estar seguros de las demandas de las tareas y de sus capacidades individuales de trabajo, decidir qué quieren obtener de cada situación, conocer qué se demanda de ellos y, en consecuencia, poner en marcha las estrategias adecuadas para lograr sus objetivos y estar preparados para cambiar de procedimiento cuando comprueben que no están alcanzando los objetivos planteados. Es por ello que no podemos olvidar la vertiente de autorregulación que implica un estudio eficaz.

En términos generales la investigación sobre la cognición del estudiante ha demostrado que tanto el conocimiento previo como el uso de estrategias cognitivas y metacognitivas juegan un papel sumamente importante en la resolución exitosa de las tareas académicas (Alexander \& Judy, 1998; Pintrich, 2004; Pintrich, Cross, Kozma \& McKeachie, 1986; Weinstein \& Mayer, 1986). En este caso, contemplamos las estrategias cognitivas como aquellos procedimientos encargados de codificar, almacenar y recuperar la información implicada en el material de estudio. Concretamente, basándonos en la propuesta de Weinstein y Mayer (1986), diferenciamos entre estrategias cognitivas de repetición (memorización), organización y elaboración: Además, incluimos también las estrategias de selección o “esencialización” que proponen Hernández y García (1991). Según Mayer (2004), las estrategias de selección, organización y elaboración de la información constituyen las condiciones cognitivas del aprendizaje significativo.

Considerando la teoría e investigación en torno a la incidencia de la implicación cognitiva sobre el rendimiento, cabe esperar que el uso de diferentes estrategias cognitivas tenga consecuencias diferentes sobre los resultados de aprendizaje. Así, podemos esperar un mejor rendimiento académico en la medida que las estrategias cognitivas implican un procesamiento informativo más significativo (e.g., Graham \& Golan, 1991; Greene \& Miller, 1996; Kardash \& Amlund, 1991; Miller, Greene, 
Montalvo, Ravindran \& Nicholls, 1996; Valle, Cabanach, Núñez, González-Pienda, Rodríguez \& Piñeiro, 2003).

Por otro lado, las estrategias de autorregulación son las encargadas de potenciar la gestión personal del proceso de aprendizaje y estudio (Weinstein \& Mayer, 1986). Sin embargo, desde nuestro punto de vista las estrategias de autorregulación promueven también el control y la supervisión, además de estos recursos propiamente cognitivos empleados para comprender información, de las posibilidades y procedimientos para, por ejemplo, buscar ayuda, decidir el empleo del tiempo, sostener unas determinadas creencias en torno a uno mismo o controlar potenciales emociones desadaptativas.

En cualquier caso, diversas investigaciones han demostrado la existencia de un conjunto de destrezas autorregulatorias altamente predictivas del éxito académico de los estudiantes (Pintrich \& DeGroot, 1990). Así, considerando el estudio como una actividad estratégica y autorregulada y tomando como referencia la diferenciación entre estrategias cognitivas y estrategias de autorregulación realizada por algunos autores (véase p.e., Pintrich \& DeGroot, 1990), en este trabajo pretendemos comprobar si existen diferencias en el uso de estos tipos estrategias en función del nivel de rendimiento académico de los estudiantes en distintas asignaturas del currículo de la enseñaza secundaria obligatoria $\left(7^{\mathrm{a}}\right.$ y $8^{\mathrm{a}}$ serie del ensino fundamental hasta el $2 .^{\circ}$ año del ensino medio en el sistema escolar brasileño).

\section{Método}

\section{Participantes}

La muestra está compuesta por 447 estudiantes de la Enseñanza Secundaria Obligatoria, (entre 12 y 16 años de edad), que cursan sus estudios en tres Institutos de Educación Secundaria de España. Del total de la muestra, 108 cursan $1^{\circ}$ ( $7^{\mathrm{a}}$ serie do ensino fundamental) (52 hombres y 56 mujeres), 148 corresponden al $2^{\circ}$ ( $8^{\mathrm{a}}$ serie do ensino fundamental) (88 hombres y 60 mujeres), 47 son estudiantes de $3^{\circ}$ ( $1 .^{\circ}$ ano do ensino médio) (27 hombres y 20 mujeres) y 144 cursan $4^{\circ}$ curso $\left(2 .^{\circ}\right.$ ano do ensino médio) (80 hombres y 64 mujeres). Del total de la muestra, el 55,3\% son hombres y el $44,7 \%$ son mujeres.

\section{Variables e Instrumentos}

En este trabajo se han evaluado tres tipos de variables: estrategias cognitivas, estrategias de autorregulación del estudio y rendimiento académico en cinco áreas curriculares.

Estrategias cognitivas. Para la evaluación de esta variable utilizamos el Cuestionario de Estrategias Cognitivas de Aprendizaje y Estudio (CECAE), elaborado por los autores de esta investigación y que evalúa las principales estrategias cognitivas (selección, organización, elaboración y memorización de información) utilizadas en las actividades de estudio y que facilitan la consecución de un aprendizaje comprensivo. Las respuestas a cada uno de los ítems del cuestionario aparecen categorizadas en una escala que va desde 1 (nunca) hasta 5 (siempre). Los índices de fiabilidad (coeficiente " $\alpha$ " de Cronbach) de cada uno de los cuatro factores, que se corresponden con las estrategias mencionadas, oscilan desde .73 para el factor "estrategias de memorización" a .82 para "estrategias de selección".

Estrategias de autorregulación. Para la evaluación de esta variable tomamos como referencia el Cuestionario de Estrategias de Control en el Estudio (ECE) de Hernández y García (1995). En este caso, sólo utilizamos dos dimensiones que aparecen contempladas en la estructura factorial del cuestionario: una destinada a la evaluación de estrategias de planificación (coeficiente " $\alpha$ " de Cronbach = 82) y otra centrada en las estrategias de supervisión-revisión (coeficiente " $\alpha$ " de Cronbach = 80) que utilizan los alumnos a la hora de estudiar. Las respuestas a cada uno de los ítems del cuestionario aparecen categorizadas en una escala que va desde 1 (nunca) hasta 5 (siempre). 
Rendimiento académico. Se suele tomar como medida del rendimiento académico las asignaturas de matemática y lengua, perdiendo la diversidad del trabajo hecho por los alumnos en las demás. En esta investigación alargamos el abanico de las asignaturas tomadas como variable dependiente de modo a aumentar el conocimiento sobre el impacto de la utilización de estrategias en el currículo. Así, para la medida del rendimiento académico recurrimos a las calificaciones académicas obtenidas por los alumnos en las siguientes asignaturas: Ciencias Naturales, Ciencias Sociales, Lengua Castellana, Lengua Extranjera, Matemáticas, Tecnología, Música, codificando los resultados de la siguiente forma: suspenso (1), aprobado (2), bien (3), notable (4) y sobresaliente (5). Posteriormente, con el fin de disponer también de una medida global del rendimiento hemos calculado el rendimiento medio en todas las asignaturas.

\section{Procedimiento}

En un primero momento, y con el fin de conocer la posible incidencia de la variable curso (ano de escolaridade) sobre la relación entre estrategias de aprendizaje-autorregulación y el rendimiento académico, se ha llevado a cabo un análisis de varianza, tomando como variable independiente el curso y como dependientes las estrategias de aprendizaje y autorregulación.

Posteriormente, el objetivo de este trabajo se ha orientado para el conocimiento de las posibles diferencias en el rendimiento académico en función del uso que hacen los estudiantes de estrategias cognitivas y de autorregulación del estudio. En este sentido, inicialmente hemos procedido al establecimiento de los grupos -según el uso de estrategias- a partir de las puntuaciones correspondientes a los percentiles 25 (baja utilización), 50 (media utilización) y 75 (alta utilización) de cada una de las estrategias cognitivas y de autorregulación del estudio. Para conocer las posibles diferencias entre los grupos recurrimos al ANOVA. Dado que cada factor está integrado por más de dos niveles o grupos, y con el fin de averiguar entre qué medias existen diferencias significativas, utilizamos como prueba de contrastes post hoc la prueba de Scheffé.

\section{Resultados}

\section{Efectos del Curso sobre el nivel de Estrategias de aprendizaje y autorregulación}

El primer objetivo consistió en comprobar si el uso de estrategias de aprendizaje y autorregulación estaba en función del año académico en que estaban los estudiantes, ya que ello podría ser una condición a tener en cuenta a la hora de abordar el objetivo principal de este estudio (relación entre la utilización de estrategias y rendimiento académico). Los datos obtenidos muestran efectos estadísticamente significativos (aunque a nivel práctico son muy pequeños), indicando que cuanto más se asciende en el curso (de primero hasta cuarto) menor es el nivel en el uso de estrategias de aprendizaje y autorregulación [selección $\left(\mathrm{F}_{3,446}=3,119 ; \mathrm{p}=, 026 ; d=, 021\right)$, elaboración $\left(\mathrm{F}_{3,446}\right.$ $=4,052 ; \mathrm{p}=, 007 ; d=, 027)$, organización $\left(\mathrm{F}_{3,446}=7,594 ; \mathrm{p}=, 000 ; d=, 049\right)$, memorización $\left(\mathrm{F}_{3,446}\right.$ $=2,842 ; \mathrm{p}=, 037 ; d=, 019)$, planificación $\left(\mathrm{F}_{3,446}=9,349 ; \mathrm{p}=, 000 ; d=, 060\right)$, supervisión $\left(\mathrm{F}_{3,446}=\right.$ $4,435 ; \mathrm{p}=, 004 ; d=, 029)]$, si bien hay que indicar que tales diferencias se obtienen únicamente para la comparación de los niveles de primero con los de cuarto curso. De hecho, el tamaño del efecto total es realmente pequeño $(d=, 035)$; no significativo en la práctica. Estos resultados concuerdan con los datos obtenidos en algunos estudios realizados con población portuguesa (Costa, 2004; Cunha, 2002; Rosário, 2004; Rosário, Trigo, Núñez, \& González-Pienda, 2005). En consecuencia, parece razonable, entonces, estimar la relación entre estrategias y rendimiento sin tener en cuenta el curso en que se encuentran los estudiantes.

\section{Estrategias cognitivas y de autorregulación y rendimiento académico}

Los resultados indican que hay diferencias estadísticamente significativas en el rendimiento obtenido en las distintas asignaturas en función de los niveles de las estrategias de selección (ver tabla 1). Sin embargo, los contrastes post hoc (prueba de Scheffé) demuestran que esas diferencias no son 
significativas entre el grupo que presenta una utilización media de estrategias medias y el grupo con utilización alta de estrategias. Además, en Música sólo hay diferencias significativas entre el grupo con baja utilización de y el grupo con elevada utilización de estrategias.

Que no existan diferencias significativas entre el grupo de alumnos con una utilización media de estrategias medias y el grupo con utilización alta de estrategias puede tener la siguiente explicación. El rango de puntuaciones del grupo de estrategias de selección medias va desde 3.57 a 4.14 y el del grupo de estrategias de selección altas va desde 4.29 a 5, con lo cual ambos grupos integrarían aquellas puntuaciones consideradas relativamente altas si tenemos en cuenta el formato de respuesta a cada uno de los ítems (desde 1 hasta 5). En este sentido, parece lógico que no haya diferencias significativas en el rendimiento académico entre un grupo que tiene unas puntuaciones en este tipo de estrategias que pueden oscilar de 3.57 a 4.14 y entre otro grupo que tiene unas puntuaciones que puede oscilar de 4.29 a 5. No obstante, con independencia de los comentarios precedentes, la tendencia general que se observa en los resultados es que cuanto más alta es la utilización de las estrategias de selección más alto es el rendimiento, y cuanto más baja es la utilización de esas estrategias también es más bajo el rendimiento.

Tabla 1 - Diferencias en rendimiento académico según los niveles de las estrategias cognitivas (selección, organización, elaboración y memorización).

\begin{tabular}{|c|c|c|c|c|c|c|c|}
\hline & \multicolumn{2}{|c|}{ Bajas } & \multicolumn{2}{|c|}{ Medias } & \multicolumn{2}{|c|}{ Altas } & \multirow[b]{2}{*}{$F(2,444)$} \\
\hline & M & DT & M & DT & M & DT & \\
\hline \multicolumn{8}{|c|}{ ESTRATEGIAS DE SELECCIÓN } \\
\hline Ciencias Naturales & 2.45 & 1.29 & 3.13 & 1.38 & 3.35 & 1.29 & $12.16 * * *$ \\
\hline Ciencias Sociales & 2.41 & 1.33 & 2.85 & 1.32 & 3.10 & 1.40 & $9.63 * * *$ \\
\hline Lengua Castellana & 2.40 & 1.17 & 3.04 & 1.27 & 3.09 & 1.35 & $13.64 * * *$ \\
\hline Lengua Extranjera & 2.17 & 1.27 & 2.76 & 1.29 & 3.01 & 1.39 & $15.27 * * *$ \\
\hline Matemáticas & 1.94 & 1.20 & 2.47 & 1.42 & 2.58 & 1.41 & $9.48 * * *$ \\
\hline Tecnología & 2.69 & 1.29 & 3.11 & 1.20 & 3.20 & 1.19 & $5.97 * *$ \\
\hline Música & 2.70 & 1.27 & 3.15 & 1.22 & 3.21 & 1.19 & $4.50 * *$ \\
\hline Rendimiento medio & 2.51 & .99 & 3.00 & .99 & 3.12 & 1.02 & $15.42 * * *$ \\
\hline \multicolumn{8}{|c|}{ ESTRATEGIAS DE ORGANIZACIÓN } \\
\hline Ciencias Naturales & 2.82 & 1.41 & 2.91 & 1.25 & 3.00 & 1.45 & .48 \\
\hline Ciencias Sociales & 2.57 & 1.33 & 2.70 & 1.31 & 2.91 & 1.46 & 2.24 \\
\hline Lengua Castellana & 2.57 & 1.23 & 2.77 & 1.31 & 3.00 & 1.32 & $4.13 *$ \\
\hline Lengua Extranjera & 2.40 & 1.34 & 2.58 & 1.26 & 2.82 & 1.44 & $3.45 *$ \\
\hline Matemáticas & 2.17 & 1.34 & 2.25 & 1.31 & 2.47 & 1.44 & 1.97 \\
\hline Tecnología & 2.71 & 1.22 & 3.01 & 1.25 & 3.17 & 1.23 & $4.72 * *$ \\
\hline Música & 2.68 & 1.24 & 3.11 & 1.20 & 3.15 & 1.29 & $3.89 *$ \\
\hline Rendimiento medio & 2.65 & 1.03 & 2.86 & .98 & 2.98 & 1.08 & $3.92 *$ \\
\hline \multicolumn{8}{|c|}{ ESTRATEGIAS DE ELABORACIÓN } \\
\hline Ciencias Naturales & 2.59 & 1.37 & 2.90 & 1.20 & 3.24 & 1.44 & $5.82 * *$ \\
\hline Ciencias Sociales & 2.37 & 1.26 & 2.65 & 1.32 & 3.23 & 1.40 & $15.90 * * *$ \\
\hline Lengua Castellana & 2.47 & 1.20 & 2.80 & 1.29 & 3.12 & 1.35 & $9.67 * * *$ \\
\hline Lengua Extranjera & 2.25 & 1.18 & 2.57 & 1.34 & 3.03 & 1.45 & $12.85 * * *$ \\
\hline Matemáticas & 2.01 & 1.23 & 2.24 & 1.32 & 2.65 & 1.47 & $8.73 * * *$ \\
\hline Tecnología & 2.71 & 1.23 & 3.02 & 1.24 & 3.20 & 1.23 & $5.34 * *$ \\
\hline
\end{tabular}


ANTONIO VALLE, SUSANA RODRÍGUEZ, RAMÓN G. CABANACH, JOSÉ C. NÚÑEZ, JULIO A. GONZÁLEZ-PIENDA Y PEDRO ROSÁRIO

\begin{tabular}{lccccccc} 
Música & 2.73 & 1.32 & 2.97 & 1.15 & 3.33 & 1.24 & $4.62 *$ \\
Rendimiento medio & 2.55 & .97 & 2.83 & 1.00 & 3.14 & 1.07 & $12.66 * * *$ \\
ESTRATEGIAS DE MEMORIZACIÓN & & & & & & \\
Ciencias Naturales & 2.50 & 1.27 & 3.06 & 1.38 & 3.15 & 1.37 & $6.20^{* *}$ \\
Ciencias Sociales & 2.43 & 1.33 & 2.85 & 1.33 & 2.90 & 1.41 & $4.93 * *$ \\
Lengua Castellana & 2.40 & 1.23 & 2.87 & 1.30 & 3.03 & 1.29 & $9.21 * * *$ \\
Lengua Extranjera & 2.23 & 1.28 & 2.67 & 1.32 & 2.84 & 1.39 & $7.81 * * *$ \\
Matemáticas & 2.08 & 1.30 & 2.39 & 1.40 & 2.39 & 1.36 & 2.33 \\
Tecnología & 2.72 & 1.31 & 3.01 & 1.16 & 3.12 & 1.23 & $3.56 *$ \\
Música & 2.50 & 1.16 & 3.24 & 1.33 & 3.13 & 1.18 & $8.59 * * *$ \\
Rendimiento medio & 2.54 & 1.01 & 2.94 & 1.01 & 2.99 & 1.03 & $8.01 * * *$ \\
${ }^{*} \mathrm{p}<.05,{ }^{* *} \mathrm{p}<.01,{ }^{* * *} \mathrm{p}<.001$ & & & & & & & \\
\hline
\end{tabular}

Con respecto a las estrategias de organización, sólo hay diferencias significativas en función de los distintos niveles de estas estrategias en Lengua Castellana, Lengua Extranjera, Tecnología, Música y rendimiento medio global (ver tabla 1). Además, los contrastes post hoc indican que las diferencias significativas mencionadas sólo se producen entre el grupo con baja utilización de estrategias de organización y el grupo con elevada utilización de estrategias de organización.

En función de estos resultados podemos deducir que se aprecian diferencias significativas en el rendimiento cuando también hay diferencias sustanciales en el uso que se hace de las estrategias de organización. Aunque, también es verdad que la tendencia de los resultados en esta estrategia va en la misma línea que en las estrategias de selección, es decir, cuanto más alta es la utilización de las estrategias de organización más alto es el rendimiento y la contraria también es verdad.

En las estrategias de elaboración se producen diferencias significativas en el rendimiento académico en las distintas asignaturas en función de los distintos niveles de este tipo de estrategias (ver tabla 1). Aunque la tendencia general de los resultados es similar a los comentados para las estrategias de selección y organización, los contrastes post hoc nos indican que no hay diferencias significativas en el rendimiento en cada una de las asignaturas entre el grupo con baja utilización de estrategias de elaboración (rango 1-3) y el grupo con media utilización de estrategias (rango 3.20-3.60), lo cual puede tener una explicación similar a otros casos comentados con anterioridad. Además, en Ciencias Naturales, Lengua Castellana, Tecnología y Música sólo se producen diferencias significativas entre el grupo con baja y el grupo con alta utilización de estrategias de elaboración.

Por lo que se refiere a las estrategias de memorización, hay diferencias significativas en el rendimiento académico en función de los distintos niveles de estas estrategias, excepto en Matemáticas (ver tabla 1). También aquí la tendencia general de los resultados es la misma que en las estrategias anteriores, aunque los contrastes post-hoc indican que no hay diferencias significativas en el rendimiento de las distintas asignaturas entre el grupo con utilización media de estrategias de memorización (rango 3.50-4) y el grupo con elevada utilización de estrategias (rango 4.25-5), lo cual tiene una explicación similar a otros resultados ya comentados. Además, en Tecnología sólo se producen diferencias significativas entre el grupo con baja utilización de estrategias de memorización y el grupo con elevada utilización de estrategias.

Con respecto a las estrategias de planificación, existen diferencias significativas en el rendimiento académico en función de los distintos niveles de este tipo de estrategias (ver tabla 2). La tendencia es la misma que en las estrategias anteriores, es decir, cuanto más elevada es la utilización de estrategias de planificación más alto es el rendimiento, y cuanto más baja es la utilización también es más bajo el rendimiento. De todas formas, los contrastes post hoc reflejan que en Ciencias Naturales no existen diferencias significativas entre el grupo con utilización media de estrategias de planificación y el grupo con elevada utilización de estrategias, mientras que en Matemáticas dichas diferencias 
no son significativas entre el grupo con baja y media utilización de estrategias. Por otro lado, en Tecnología sólo hay diferencias significativas entre el grupo con baja y el grupo con alta utilización de estrategias de planificación.

Tabla 2 - Diferencias en rendimiento académico según los niveles de las estrategias de autorregulación (planificación y supervisión-revisión).

\begin{tabular}{|c|c|c|c|c|c|c|c|}
\hline & \multicolumn{2}{|c|}{ Bajas } & \multicolumn{2}{|c|}{ Medias } & \multicolumn{2}{|c|}{ Altas } & \multirow[b]{2}{*}{$\mathrm{F}(2,444)$} \\
\hline & M & DT & M & DT & M & DT & \\
\hline \multicolumn{8}{|c|}{ ESTRATEGIAS DE PLANIFICACIÓN } \\
\hline Ciencias Naturales & 2.32 & 1.33 & 3.00 & 1.27 & 3.30 & 1.38 & $12.80 * * *$ \\
\hline Ciencias Sociales & 2.25 & 1.24 & 2.69 & 1.33 & 3.27 & 1.38 & $20.24 * * *$ \\
\hline Lengua Castellana & 2.28 & 1.20 & 2.87 & 1.23 & 3.22 & 1.30 & $19.60 * * *$ \\
\hline Lengua Extranjera & 2.16 & 1.31 & 2.57 & 1.27 & 3.10 & 1.38 & $17.30 * * *$ \\
\hline Matemáticas & 1.90 & 1.22 & 2.24 & 1.31 & 2.75 & 1.43 & $14.20 * * *$ \\
\hline Tecnología & 2.61 & 1.20 & 2.97 & 1.26 & 3.30 & 1.18 & $9.85 * * *$ \\
\hline Música & 2.50 & 1.27 & 2.98 & 1.18 & 3.52 & 1.17 & $13.27 * * *$ \\
\hline Rendimiento medio & 2.43 & .99 & 2.84 & .97 & 3.22 & 1.03 & $21.73 * * *$ \\
\hline \multicolumn{8}{|c|}{ ESTRATEGIAS DE SUPERVISIÓN-REVISIÓN } \\
\hline Ciencias Naturales & 2.35 & 1.25 & 3.04 & 1.36 & 3.33 & 1.34 & $13.86 * * *$ \\
\hline Ciencias Sociales & 2.19 & 1.22 & 2.97 & 1.36 & 3.02 & 1.39 & $17.29 * * *$ \\
\hline Lengua Castellana & 2.25 & 1.16 & 2.95 & 1.33 & 3.18 & 1.24 & $20.84 * * *$ \\
\hline Lengua Extranjera & 2.18 & 1.28 & 2.68 & 1.37 & 2.95 & 1.33 & $11.98 * * *$ \\
\hline Matemáticas & 1.90 & 1.17 & 2.46 & 1.45 & 2.52 & 1.36 & $9.12 * * *$ \\
\hline Tecnología & 2.54 & 1.17 & 3.25 & 1.27 & 3.09 & 1.19 & $12.61 * * *$ \\
\hline Música & 2.56 & 1.26 & 3.03 & 1.22 & 3.42 & 1.15 & $9.98 * * *$ \\
\hline Rendimiento medio & 2.38 & .96 & 3.00 & 1.04 & 3.11 & .97 & $22.67 * * *$ \\
\hline${ }^{*} \mathrm{p}<.05, * * \mathrm{p}<.01$, & & & & & & & \\
\hline
\end{tabular}

En cuanto a las estrategias de supervisión-revisión se producen diferencias significativas en el rendimiento académico de cada una de las asignaturas en función de los distintos niveles de estas estrategias (ver tabla 2). Del mismo modo que en las estrategias anteriores, la tendencia general de los resultados es la misma, cuanto más elevada es la utilización de estrategias más alto es el rendimiento y cuanto más baja es la utilización de estrategias más bajo es el rendimiento. De todas formas, igual que sucedía en otros resultados ya comentados, los contrastes post-hoc indican que no hay diferencias significativas en el rendimiento académico de cada una de las asignaturas entre el grupo con utilización media (rango 3.33-4) y alta (rango 4.17-5) de estrategias de supervisión-revisión.

\section{Discusión}

El aprendizaje autorregulado se ha convertido en un concepto fundamental tanto en las investigaciones como en la práctica educativa (Pintrich, 2000, 2004; Rosário, Núñez \& González-Pienda, 2006), pues ofrece respuesta a las necesidades psicoeducativas que implican formar personas capaces de adoptar una considerable autonomía en su formación y que desarrollen una serie de herramientas que les permitan un aprendizaje continuo, más allá de su vida académica. Supone también un concepto unificador, ya que relaciona diferentes campos de investigación (e.g., cognición, estrategias de aprendizaje, motivación, etc.) que juntos proporcionan un cuadro coherente sobre cómo un 
estudiante gestiona las complejas actividades inherentes al aprendizaje académico. Además, todas estas competencias de autorregulación constituyen un factor crítico para el aprendizaje y rendimiento académico de los estudiantes (Paris \& Paris, 2001; Pintrich \& Schrauben, 1992; Rosário, 2004; Rosário, Núñez \& González-Pienda, 2004; Schunk \& Zimmerman, 1998). Así, al posibilitar un aprendizaje autónomo y permanente, estas competencias en autorregulación posibilitan que los estudiantes puedan aumentar su rendimiento y éxito académico a través del uso de diferentes estrategias, controlar y regular muchas aspectos de su cognición, motivación, y conducta, seleccionar y estructurar ambientes de aprendizaje, mediando entre las características contextuales y personales, y fijarse metas y controlar su cumplimento (Núñez, et al., 2006).

En nuestro estudio, las diferencias en el rendimiento académico en función de los distintos niveles de utilización de las estrategias cognitivas y de autorregulación del estudio demuestran que, en general, una mayor utilización de las diferentes estrategias suele traer consecuencias positivas en la mejora del rendimiento en las distintas asignaturas y, complementariamente, una menor utilización de estas estrategias suele estar asociado con los niveles de rendimiento más bajos. En consonancia con resultados de investigaciones previas, el uso de estrategias cognitivas que implican un procesamiento profundo de la información mantiene una relación positiva y significativa con el rendimiento de los estudiantes en diferentes materias académicas (Greene, Miller, Crowson, Duke \& Akey, 2004; Valle, Cabanach, Rodríguez, Núñez, J.C. \& González-Pienda, 2006).

Por otra parte, también parece evidente que las estrategias de autorregulación del estudio (planificación y supervisión-revisión) tienen una relación mucho más potente con el rendimiento académico en las distintas asignaturas y, probablemente, sean más determinantes que las estrategias cognitivas en la nota final. Estos resultados coinciden con las aportaciones de otros estudios (e.g., Almeida, Canelas, Rosário, Núñez, \& González-Pienda, 2005; Pintrich \& De Groot, 1990), no sólo en cuanto a la relación entre estrategias cognitivas y rendimiento académico, sino también en resaltar que dicha relación es mucho más fuerte en el caso de las estrategias de autorregulación. Esto pone de manifiesto la importancia de que los estudiantes no sólo deben comprender qué estrategias cognitivas utilizar, sino también cómo y cuándo utilizarlas (Brown, Bransford, Campione \& Ferrara, 1983; Paris, Lipson \& Wixson, 1983; Pressley, 1986). Esta actuación estratégica a nivel académico es la que puede garantizar mejoras significativas en el rendimiento académico de las diferentes asignaturas en la medida que los profesores en clase trabajen con los alumnos el conocimiento declarativo, procedimental y condicional de las estrategias de aprendizaje, enseñando explicita e intencionalmente un largo abanico de estrategias y practicándolas en el contexto específico de las distintas asignaturas. Estos procedimientos instructivos están orientados para promover la utilidad percibida de estas estrategias y su trasferencia para distintas tareas y contenidos de aprendizaje. En suma, habilitando los alumnos para aprender a aprender y, así, promover su éxito escolar.

En realidad, a pesar de que las leyes educativas vigentes y los resultados de la investigación, refuerzan la importancia de que los estudiantes aprendan a autorregular su aprendizaje a partir de una enseñanza sistemática e intencional; de hecho pocos profesores preparan a sus alumnos con esas capacidades que les conduzcan a poder desempeñar un aprendizaje personal y de manera significativa (Rosário et al., 2006; Zimmerman, 2002).

El proceso que lleva al aprendizaje significativo, de acuerdo con Mayer (2004) depende tanto de la forma en cómo procesa el aprendiz la información (es decir, del lado del aprendizaje) como del material que se presenta (es decir, del lado de la enseñanza). Por tanto, disponemos de dos formas complementarias de promover el aprendizaje significativo: mejorando la forma en que los estudiantes procesan la información (es decir, las estrategias de aprendizaje y de autorregulación) y mejorando la forma en que se presentan los materiales (es decir, los métodos instruccionales).

Generalmente, a los profesores se les ha pedido que incorporen a sus aulas todo un conjunto de innovaciones desarrolladas fuera de éstas. Por otra parte, la formación tradicional de los profesores ha considerado a los profesores como "alumnos pasivos” que llevarán a cabo la incorporación de innovaciones mediante el masivo entrenamiento técnico y la ayuda de los investigadores. En realidad, posiblemente existan muy pocos programas de formación de profesores que fomenten la idea de los 
profesores como innovadores en sí mismos. En estos casos, se prestará mayor atención al desarrollo de las habilidades de autorregulación en los propios profesores y se les invitará a convertirse en profesores-investigadores (Lieberman, 1995; Corno \& Randi, 2000). Por tanto, la gran cantidad de investigación desarrollada sobre el aprendizaje autorregulado de los alumnos, junto con la nueva forma de entender el modo en que los profesores desarrollan su tarea educativa, constituyen las bases para la investigación sobre el aprendizaje autorregulado en los profesores. Esta nueva línea de investigación es, ahora, aún más pertinente para poder responder a las demandas del proceso de Bolonia que está en curso en las Universidades europeas y que implica equipar a los alumnos para responder con más autonomía y autorregulación a las exigencias del contexto académico.

Pero, como dice el dicho popular, "nadie da lo que no tiene”; por eso es urgente capacitar los profesores para esta nueva tarea educativa.

\section{Referencias}

Alexander, P., \& Judy, J. (1988). The interaction of domain-specific and strategic knowledge in academic performance. Review of Educational Research, 58, 375-404

Almeida, L. S., Canelas, C., Rosário, P., Núñez, J. C., \& González-Pienda, J.A. (2005). Métodos de estudo e rendimento escolar: sua relação em alunos do Ensino Secundário Português. Revista de Educação. 12, 63-74.

Brown, A.L., Bransford, J.D., Campione, J.C., \& Ferrara, R.A. (1983). Learning, remembering and understanding. En J. Flavell \& E. Markman (Eds.), Handbook of child psychology: Vol. 3. Cognitive development (pp. 515-629). New York: Wiley.

Corno, L., \& Randi, J. (2000). ¿Una teoría del diseño educativo basado en el aprendizaje autorregulado? En Ch. M. Reigeluth (Ed.), Diseño de la instrucción. Teorías y modelos. Vol. 1 (pp. 305-334). Madrid: Aula XXI. Santillana.

Costa, P. (2005). Estratégias de auto-regulação na aprendizagem em História: Estudo no $2 .{ }^{\circ}$ CEB, Supervisão no ensino da História. Tese de Mestrado. Braga: Universidade do Minho.

Cunha, A. (2002). Aprendizagem auto-(des)regulada? Rotas e percursos em alunos dos $2 .^{\circ}$ e 3. ${ }^{\circ}$ Ciclos do Ensino Básico. Tese de Mestrado. Braga: Universidade do Minho.

Graham, S., \& Golan, S. (1991). Motivational influences on cognition: Task involvement, ego involvement and depth of information processing. Journal of Educational Psychology, 83, 187194

Greene, B.A., \& Miller, R.B. (1996). Influences on course performance: Goals, perceived ability, and self-regulation. Contemporary Educational Psychology, 21, 181-192

Greene, B.A., Miller, R.B., Crowson, H.M., Duke, B.L., \& Akey, K.L. (2004). Predicting high school students' cognitive engagement and achievement: contributions of classroom perceptions and motivation. Contemporary Educational Psychology, 29, 462-482

Hernández, P., \& García, L.A. (1991). Psicología y enseñanza del estudio. Teorías y técnicas para potenciar las habilidades intelectuales. Madrid: Pirámide.

Hernandez, P., \& García, L.A. (1995). Cuestionario de Estrategias de Control en el Estudio (ECE). Departamento de Psicología Educativa, Evolutiva y Psicobiología. Universidad de La Laguna.

Kardash, C.M., \& Amlund, J.T. (1991). Self-reported learning strategies and learning from expository text. Contemporary Educational Psychology, 16, 117-138

Lieberman, A. (1995). Practices that support teacher development: Transforming conceptions of professional learning. Phi Delta Kappan, 76, 591-596.

Mayer, R. E. (2004). Psicología de la educación. Madrid: Pearson Educación. 
Miller, R.B., Greene, B.A., Montalvo, G.P., Ravindran, B., \& Nicholls, J.D. (1996). Engagement in academic work: The role of learning goals, future consequences, pleasing others, and perceived ability. Contemporary Educational Psychology, 21, 388-442.

Núñez, J. C., Solano, P., González-Pienda, J. A., \& Rosário, P. (2006). El aprendizaje autorregulado como medio y meta de la educación. Papeles del Psicólogo, 27, 139-147.

Paris, S. G., \& Paris, A. H. (2001). Classroom application of research on self-regulated learning. Educational Psychologist, 36, 89-101.

Paris, S.G., Lipson, M.Y., \& Wixson, K. (1983). Becoming a strategic reader. Contemporary Educational Psychology, 8, 293-316.

Pintrich, P. (2004). A conceptual framework for assessing motivation and self-regulated learning in college students. Educational Psychology Review, 16, 385-407.

Pintrich, P. R., \& Schrauben, B. (1992). Students' motivational beliefs and their cognitive engagement in classroom tasks. En D. Schunk \& Meece (Eds.), Student perceptions in the classroom: Causes and consequences (pp. 149-183). Hillsdale, NJ: Erlbaum.

Pintrich, P.R. (2000). The role of goal orientation in self-regulated learning. En M. Boekaerts, P.R. Pintrich \& M. Zeidner (Eds.), Handbook of self-regulation (pp. 451-502). Academic Press.

Pintrich, P.R., \& DeGroot, E.V. (1990). Motivational and self-regulated learning componentes of classroom academic performance. Journal of Educational Psychology, 82, 33-40

Pintrich, P.R., Gross, D.R., Kozman, R.B., \& McKeachie, W.J. (1986). Instructional Psychology. Annual Review of Psychology, 37, 611-651

Pressley, M. (1986). The relevance of the good strategy user model to the teaching of mathematics. Educational Psychology, 21, 139-161.

Rosário, P. (2004). Estudar o Estudar: As (Des)venturas do Testas. Porto: Porto Editora.

Rosário, P., Mourão, R., Núnez, J. C., González-Pienda, J.A. Solano, P. \& Valle, A. (2007). Eficacia de un programa instruccional para la mejora de procesos y estrategias de aprendizaje en la enseñanza superior. Psicothema, 19, 353-358.

Rosário, P., Mourão, R., Soares, S., Chaleta, E., Grácio, L., Núnez, J. C., \& González-Pienda, J. (2005). Trabalho de casa, tarefas escolares, auto-regulação e envolvimento parental. Psicologia em Estudo, 10, 343-351.

Rosário, P., Núñez, J. C., \& González-Pienda, J. (2006). Cartas do Gervásio ao seu Umbigo. Comprometer-se com o Estudar na Universidade. Coimbra: Almedina Editores.

Rosário, P., Núñez, J., \& González-Pienda, J. (2004). Stories that show how to study and how to learn: an experience in Portuguese school system. Electronic Journal of Research in Educational Psychology, 1, 131-144.

Rosário, P., Trigo, J., Núnez, J. C., \& González-Pienda, J. (2005). SRL Enhancing Narratives: Testas’ (Mis)adventures. Academic Exchange Quarterly, Winter, 9, 73 -77.

Schunk, D. H., \& Zimmerman, B. J. (1998). Conclusions and future directions for academic interventions. En D. H. Schunk y B. J. Zimmerman (Eds.), Self-Regulated learning. From teaching to self-Reflective Practice (pp. 225-234). Hillsdale, NJ: Lawrence Erlbaum Associates, Inc.

Valle, A., Cabanach, R.G., Núñez, J.C., González-Pienda, J.A., Rodríguez, S., \& Piñeiro, I. (2003). Cognitive, motivational, and volitional dimensions of learning: An empirical test of a hypothetical model. Research in Higher Education, 44, 557-580. 
Valle, A., Cabanach, R.G., Rodríguez, S., Núñez, J.C., \& González-Pienda, J.A. (2006). Metas académicas, estrategias cognitivas y estrategias de autorregulación del estudio. Psicothema, 18, 165-170.

Weinstein, C.E., \& Mayer, R.E. (1986). The teaching of learning strategies. En M.C. Wittrock (Ed.), Handbook of research on teaching (pp. 315-327). New York: McMillan.

Zimmerman, B. J. (2002). Becoming a self-regulated learner: an overview. Theory into Practice, 41, 64-70. 